\title{
Post- infectious bronchiolitis obliterans in children: clinical and radiological evaluation and lon- term results
}

\author{
Hakan Yazan ${ }^{1}$, Fathouma Khalif ${ }^{1}$, Lina Al Shadfan ${ }^{2}$, Sabriye Bilgin ${ }^{3}$, Mustafa Nursoy ${ }^{4}$, \\ Fatma Cakir ${ }^{2}$, Zeynep Cakin ${ }^{4}$, Selcuk Uzuner ${ }^{5}$, and Erkan Cakir ${ }^{2}$ \\ ${ }^{1}$ Bezmialem Vakif Universitesi Tip Fakultesi \\ ${ }^{2}$ Bezmialem Vakif University \\ ${ }^{3}$ Istanbul Medipol University Faculty of Medicine \\ ${ }^{4}$ Bezmialem Vakıf Üniversitesi \\ ${ }^{5}$ Bezmialem Vakif Universitesi
}

October 14, 2020

\begin{abstract}
Abstract Objective: This study aims to evaluate clinical and radiological findings and treatment outcomes of the patients with PIBO. Methods: One hundred fourteen children were enrolled. Initial demographic and clinical findings were evaluated. Pre- and post-treatment symptoms, radiological findings and scores, the number of admissions to the hospital and PICU were compared. Results: Seventy-three patients were male. The median age of the patients at initial pulmonary injury was 7.2 months, the median age at diagnosis was 17.5 months and the median interval from initial injury to diagnosis was 8.5 months. Thirty-five patients had mechanical ventilation history. Persistent wheezing was the most common complaints. The most common radiological findings were peribronchial thickening and air-trapping. Sixty-eight patients were treated with inhaled corticosteroid (IC), systemic corticosteroid (SC) and azithromycin (AZT) combination. 25 patients were treated with only IC, 14 with IC and AZT, and 7 with IC and SC. $82,5 \%$ of patients had clinical improvement. Chronic respiratory failure occurred in 11 patients, and three patients died. Bronchiectasis, atelectasis, hyperinflation and air trapping in HRCT improved significantly. Bhalla scores decreased from 8.3 to $6.5(\mathrm{p}=0,001)$. IC, SC and AZT combination was effective than the other treatments. Conclusions: This study is one of the largest studies in the literature and one of the few studies that evaluate clinical and radiological outcomes. We found that IC, AZT and SC combination is clinically and radiologically effective in patients with PIBO.
\end{abstract}

\section{Introduction}

Post-infectious bronchiolitis obliterans (PIBO) is characterized by persistent obstructive symptoms and radiological findings after infectious lung injury. ${ }^{1}$ The prevalence of the disease is unknown, but PIBO is more common in children than adults. ${ }^{2}$ PIBO always occurs after a severe lower tract infection. Adenovirus, influenza, respiratory syncytial virus, rubeola and mycoplasma pneumonia have been shown to be associated with PIBO. Adenovirus is the most common infectious agent that causes PIBO. ${ }^{3}$ Inflammation and airway obliteration were demonstrated in pathological studies of the lung. ${ }^{4}$ Persistent cough, wheezing, sputum production, dyspnea are the most common symptoms of PIBO. ${ }^{5}$ Although the histopathological examination is the gold standard for PIBO, it is not routinely used because of the multifocal pattern of the disease and the risks of biopsy procedure. ${ }^{6}$ PIBO is diagnosed by clinical history, typical radiological findings and the exclusion of the other diseases that may cause chronic lung diseases like severe asthma, bronchopulmonary dysplasia, cystic fibrosis, tuberculosis, and immunodeficiency. ${ }^{7}$ Pulmonary function test (PFT) and highresolution computed tomography (HRCT) are important tools for diagnosis. Obstructive pattern is present 
in PFT and air trapping, peribronchial thickening, hyperinflation, and bronchiectasis are the radiological findings of PIBO. ${ }^{8}$ There is no universal standard therapy in PIBO. Inhaled corticosteroid (IC), systemic corticosteroid (SC), pulse steroid, azithromycin (AZT), and immunoglobulin are used for the treatment of PIBO.${ }^{9}$ There are a few studies about PIBO in children. However, to our knowledge, there has been no published data yet to evaluate treatment efficacy by clinical and radiological comparison.

The present study aims to establish demographic, initial clinical and radiological characteristics, treatment status of the patients and to compare pre- and post-treatment clinical status and radiological findings. To our knowledge, the present study was one of the largest series of patients with PIBO and the first study to compare radiological scores pre- and post-treatment.

\section{Materials and Methods}

One hundred and fourteen children with PIBO who were followed up at the Pediatric Pulmonology Department of Bezmialem Vakif University between 2011-2020 were included in this study.

Diagnosis

Diagnosis of PIBO was made according to clinical criteria: I: History of an acute and severe lower respiratory infection in a previously healthy child. II: Evidence of persistent airway obstruction after the acute event, identified by physical examination or PFT. This obstruction is unresponsive to at least a two-week course of systemic steroid and bronchodilators. III: Chest radiographic findings of obstructive lung disease, such as hyperinflation, atelectasis, airway wall thickening and bronchiectasis. IV: Mosaic pattern and air trapping in HRCT. V: Exclusion of other chronic lung diseases that progress with permanent respiratory symptoms, such as tuberculosis, cystic fibrosis, bronchopulmonary dysplasia, immunodeficiencies, severe asthma and alpha 1 antitrypsin deficiency.

Sweat test, immunoglobulin levels, echocardiography, microbiological evaluation, HRCT, and total IgE were performed in all patients. Skin prick test and bronchoscopy were performed to selected patients for differential diagnosis. PFT was applied to children above six years ${ }^{7}$.

Demographic and clinical characteristic findings

Gender, gestational age, age at the beginning of persistent complaints, age of diagnosis, the interval from initial injury to the age of diagnosis, follow-up duration, family history of asthma, smoking exposure, number of admission to the hospital and pediatric intensive care unit (PICU), history of mechanical ventilation, current complaints, current physical examination findings and initial PFT results were evaluated retrospectively.

Radiological examination

All HRCT scans were evaluated by the same radiologist who was unaware of the clinical condition of the patients. The Bhalla scoring system was applied to evaluate HRCT findings. This scoring system evaluates the followings: (1) severity of bronchiectasis; (2) extent of bronchiectasis; (3) peribronchial thickening; (4) extent of mucus plugs; (5) abscesses/sacculation; (6) generality of bronchial division involvement; (7) number of bubbles; (8) emphysema; and (9) collapse/consolidation. Scores from 0 to 3 were assigned to each of the first seven categories (severity of bronchiectasis, the extent of bronchiectasis, peribronchial thickening, the extent of mucus plugs, abscesses/sacculation, the generality of bronchial division involvement, and the number of bubbles) and from 0 to 2 for the last two categories (emphysema and collapse/consolidation). The total score had a maximum value of 25 and was calculated by adding individual scores for each item. The scores were classified as Grade 0 (none), Grade I (mild,1-9), Grade II (moderate,10-14) and Grade III (severe,15-18).

\section{Treatment}

Treatments of the patients were evaluated retrospectively. Patients with PIBO are referred to our clinic in two different ways (Figure 1). 1- Patients with a referral to our clinic within six months after initial 
pulmonary injury and have active complaints and typical radiological findings. 2- Patients with a referral to our clinic longer than six months after initial lung injury.

Patients in the first way were routinely administered inhaled steroid (IC), systemic corticosteroid (SC), and azithromycin (AZT) combination because of having marked clinical and radiological findings and they were in the early stage of the disease (Treatment group 1).

Patients in the second way are divided into four treatment groups. Patients who had severe clinical symptoms and radiological findings were treated with IC, SC and AZT (Treatment group 1), patients with mild clinical symptoms and radiological findings were administered only IC (Treatment group 2), patients with moderate clinical symptoms and radiological findings with bronchiectasis were treated with IC and AZT (Treatment group 3) and patients with moderate clinical symptoms and radiological findings without bronchiectasis were treated using IC and SC (Treatment group 4). Total number of patients were: treatment group-1: 68 $(59,7 \%)$, treatment group-2: $25(21,9 \%)$, treatment group-3: $14(12,3 \%)$ and treatment group-4: $7(6,1 \%)$ (Table 1).

AZT was administered $10 \mathrm{mg}$ per $\mathrm{kg}$ three times a week for six months. The dose of $\mathrm{SC}$ was $2 \mathrm{mg} / \mathrm{kg} / \mathrm{day}$ for four weeks and the dose was gradually decreased to $0,5 \mathrm{~m} / \mathrm{kg}$ /day and continued for six months with minimal dose.

Post-treatment evaluation of clinical findings and pulmonary function tests

Pre- and post-treatment clinical findings were evaluated. Clinical findings, number of hospitalization, number of PICU admission, oxygen requirements were compared.

Thirty-four patients were able to do PFT after treatment. Forced vital capacity (FVC), forced expiratory volume in one second (FEV1) and FEV1/FVC ratio were recorded. Post-treatment results of 22 patients who could perform plethysmography and diffusing capacity of the lung for carbon monoxide (DLCO) were also evaluated. Tidal volume, total lung capacity, residual volume, functional residual capacity and diffusing capacity were evaluated in these patients.

Pre- and post-treatment radiological comparison of the patients

While all 114 of patients had initial HRCT, 81 patients had post-treatment HRCT. Post-treatment HRCT did not yield in the remaining 33 patients because their parents did not allow. Pre- and post-treatment radiological findings and Bhalla scores were compared in these 81 patients.

All statistical analyses were performed using SPSS Statistics vs. 22.0. Numeric indicators were considered as mean, standard deviation, median, minimum, maximum and interquartile range. Two groups were compared using Fishers exact test and $\chi^{2}$ test. Paired sample $t$-test and Wilcoxon test were utilized to compare paired groups. A $p$-value $<0,05$ was considered to indicate statistical significance.

This study was approved by the Ethics Committee of Bezmialem Vakif University. Informed consent was obtained from patients and their parents.

\section{Results}

Initial demographic, clinical and radiological findings

One hundred fourteen patients with PIBO were enrolled in this study. Initial demographic, clinical and radiological findings of the patients are presented in Table 1. Seventy-four patients were male. The current median age was 73,5 months. The median age at initial pulmonary injury was 7,2 months. The median age at diagnosis was 17.5 months and the median interval from initial injury to diagnosis was 8,5 months. Twenty-one $(18,4 \%)$ patients had a history of asthma. Asthma history in family and smoking exposure were positive in almost half of the patients. 103/114 patients had a history of hospital admission at least once with a diagnosis of lower respiratory tract infection. One-third of the patients had a history of PICU and mechanical ventilation. The most common symptoms are persistent cough, persistent wheezing, sputum and shortness of breath. In initial HRCT (n:114), peribronchial thickening was detected in all and air trapping 
existed over ninety percent. About half of the patients had bronchiectasis. Initial PFT was performed to only 17 patients because of their younger age. Mean of FEV1 and FVC of initial PFTs were 59,7 $\pm 29,1$ and $61,9 \pm 28,2$, respectively. Flexible fiberoptic bronchoscopy was performed in 75 patients. Serous secretions were obtained in $75 \%$ of bronchoalveolar lavage specimen. Bacterial, viral and fungal cultures of bronchoalveolar lavage revealed no isolation. Viral etiological agent could be detected in only five patients. This is because most patients were referred to our hospital for a long time after the acute injury (Table 1).

Post-treatment evaluation of clinical findings and pulmonary function tests

Clinical evaluation and PFT results of the patients after treatment are presented in Table 2. None of the patients required admittance to PICU after treatment. Hospitalization rates with lower respiratory tract infections decreased from $90,3 \%$ to $12,3 \%$. The symptoms and signs improved in more than $80 \%$ of the patients. However, they persisted in approximately $20 \%$ of the patients despite treatment. Chronic respiratory failure occurred in 11 patients, and all of them are on non-invasive ventilation therapy.

PFT was performed to 34 patients after treatment. Approximately $20 \%$ of the patients had severe and moderate decline both in FEV1 and FVC (Table 2).

Post-treatment plethysmography was performed in 22 patients (Table 2). Total lung capacity and functional residual capacity increased in most of the patients. DLCO decreased moderately and severely in $20 \%$ of patients (Table 2).

Forty-six patients $(40,4 \%)$ are still under treatment with IC and 24 patients $(21,6 \%)$ under IC and long-acting beta-agonist.

Pre and post-treatment radiological comparison of the patients

Improvement was observed in all radiological findings except peribronchial thickening (Table 3). There was a significant difference between pre- and post-treatment Bhalla scores of the patients $(\mathrm{p}<0,01)$. Among treatment subgroups, radiological findings and Bhalla scores were compared (Table 4). Treatment group 1 had a significant improvement in both radiological findings and Bhalla scores. There was a radiological improvement in the other treatment groups but not statistically significant due to the low number of patients in these groups.

\section{Discussion}

PIBO is a form of chronic obstructive lung disease that arises from severe acute lower tract infection. In this study, clinical and radiological findings and treatment outcomes and long-term follow-up results are evaluated.

In our study, the male gender was dominant like in the literature. ${ }^{10,11}$ Initial pulmonary injury that causes PIBO generally occurs during the first two years of life. ${ }^{12,13}$ Median age at the initial injury of our patients was 7,2 months and the median age of diagnosis was 17,5 months. There was a median 8,5 months interval from initial injury until diagnosis in our study. A study from South Korea noted a similar duration in the interval as our study (Mean eight months, Min-Max 2-24 months). ${ }^{14}$ Bo Qia et al. reported a median of three months interval (Min-Max 1-43 month). ${ }^{15}$ Another study also showed that PIBO was diagnosed on an average of two years (Min- Max 0,2-7,6 years) after acute illness. ${ }^{16}$

PIBO and severe asthma are often difficult to be distunguished. Some studies suggested that atopy could be associated with PIBO. ${ }^{17,18,19,20}$ Similarly, in our study, approximately $20 \%$ of our patients had atopy.

PIBO usually occurs after severe acute lower respiratory infection. The number and duration of hospitalization increased in patients with PIBO. ${ }^{21,22}$ Colom et al. noted mechanical ventilation as a strong risk factor for developing PIBO after adenovirus infection. ${ }^{23}$ In the literature, mechanical ventilation percentages ranged from $23 \%$ to $52,5 \% .{ }^{24,25}$ In our study, $90,3 \%$ of patients required hospitalization at least once and one-third of the patients had a history of mechanical ventilation. 
Clinical findings in PIBO include persistent cough, sputum, wheezing, and shortness of breath. ${ }^{26}$ Our patients' findings were consistent with other studies and persistent cough and wheezing are the most common findings in our study. ${ }^{24,27}$

PIBO generally occurs after a severe viral infection. ${ }^{8}$ In our study, there were only five proven viral agents. Because our hospital is a tertiary center, most patients are referred from other hospitals within weeks or months long after the acute initial injury. Thus, it was not possible to determine etiological agents.

The diagnosis of PIBO is made by clinical and radiological evaluation and the exclusion of other respiratory diseases. ${ }^{7}$ Sweat chloride test, skin prick test, tuberculin skin test, immunoglobulin levels and echocardiography were performed in our patients for differential diagnosis, and all of them were normal. Ya-nan- li et al. performed a bronchoscopy on half of the patients in their series, and they found bronchial mucosal inflammations in 12 patients, tracheomalacia in two patients and normal findings in six patients. ${ }^{10}$ Among our 114 patients, bronchoscopy was performed in 75 patients and seros secretions were mostly seen.

Although HRCT is the most sensitive tool for diagnosis, there are no pathognomonic radiological findings in patients with PIBO. ${ }^{3}$ Air trapping, peribronchial thickening, bronchiectasis, atelectasis and mucus plugs are mostly seen in patients with PIBO. ${ }^{7}$ Peribronchial thickening, air trapping and bronchiectasis were also the most common radiological findings in our study. Cazzata et al. and Bo Qio et al. found similar findings with our study. ${ }^{28,15}$ Bronchiectasis rates may vary in a considerable range in the literature. ${ }^{10,29,30}$ In our study, there was bronchiectasis in about half of the patients in HRCT.

Since PIBO is a disease affecting smaller airways, the obstructive pattern is mostly present in PFTs. ${ }^{9}$ Because of the young age of our study, initial PFT could only be performed in 17 patients. Moderate and severe obstruction in FEV1 and FVC were found. Our FEV1 and FVC results were concomitant with other studies. ${ }^{25,29}$ There were studies that noted lower and higher FEV1/FVC values than our study. ${ }^{28,31-33}$

Treatment of PIBO is variable, and there is no standard protocol in the literature. Zhang et al. administered inhaled budesonide, inhaled ipratropium bromide and inhaled terbutaline to their patients for one year and found an improvement of $86,6 \%$ in symptom scores and 56,6\% in PFT (27). In a study in which azithromycin and prednisolone were administered for a long time, improvement of the clinical findings was shown in $62,5 \%$ of their patients, but no radiological improvement was established. ${ }^{19}$ In another study, after six months of azithromycin and oral steroid combination therapy, the symptoms regressed in $85,7 \%$ of patients. ${ }^{10}$ Another study from Turkey showed that systemic steroid therapy in PIBO did not lead to a satisfactory clinical outcome in their patients. ${ }^{34}$ We administered four different treatment regimes to the patients. After treatment, we found significant improvement in clinical findings. Hospital admission rate of our patients decreased from 90,3\% to $12,3 \%$. There was no PICU admission requirement after treatment. We also evaluated radiological findings of pre- and post-treatment of patients with PIBO. There was a significant improvement in bronchiectasis, atelectasis, hyperinflation and air trapping findings. Wang et al. showed that HRCT observation in $62,5 \%$ of their patients was significantly improved. ${ }^{19}$ We also detected a significant decrease Bhalla score pre- and post-treatment. Bhalla score is actually created for bronchiectasis. The studies in the literature are mostly concerning CF and non-CF bronchiectasis; there are a few studies that evaluated Bhalla score in non-bronchiectasis patients. A study that evaluated the Bhalla score in PIBO found that mostly patients of their $(90,5 \%)$ had moderate Bhalla score; however, to our knowledge, there is no evaluation of pre- and post-treatment in this study. ${ }^{31}$ In our study, $66,3 \%$ of patients had mild Bhalla score and $27,4 \%$ of patients had moderate Bhalla score. We noted that post-treatment mean Bhalla scores significantly decreased. To our knowledge, this study is the first study in the literature that evaluated the efficiency of treatment by a radiological scoring. Radiological comparison of treatment subgroups indicated combined IC, OC and AZT therapy (Therapy 1) improved radiological findings and increased Bhalla scores significantly; only IC treatment (Therapy 2) increased Bhalla scores significantly.

Plethysmography is a lung function test that shows lung volumes and used to detect hyperinflation in patients with PIBO. There was no pre-treatment plethysmography study in our patients. The results of 22 patients who were able to perform plethysmography after treatment demonstrated that hyperinflation exists in our 
patients. Plethysmography findings of our patients were consistent with other studies in the literature (35, 31). DLCO is a measurement that shows the transfer of carbon monoxide from the alveolar membrane to the blood. In our study, the results of 22 patients revealed a mild reduction in DLCO. The results of the few studies that evaluate DLCO in PIBO are consistent with our study. ${ }^{8}$

Among all our patients who followed up a median of 48 months, twelve patients had oxygen requirements. Chronic respiratory failure developed in eleven patients $(9,6 \%)$ and three patients $(3,6)$ died. In a prospective study that evaluated 58 patients, oxygen requirement was revealed in $19 \%$ of the patients. ${ }^{35}$

In conclusion, the current study is one of the largest numbers of patients with PIBO and one of a few studies that evaluate clinical and radiological features pre- and post-treatment. Also, Current study is one of the studies in which PIBO patients were followed for a long time. We found that azithromycin and oral corticosteroid treatment are clinically and radiologically effective in patients with PIBO. However, prospective randomized-controlled studies are needed to prove this.

There were a few limitations in our study. First, because of the young age of our patients, the number of patients was able to perform PFT were few, second, we have no control groups in treatment, and finally, we had fewer patients who had microbiological agent etilogy.

Acknowledgment

No conflict of interest was declared by authors.

Figure legend

Figure 1. Treatment flow chart of the patients

\section{References}

1. Frohlic LF, Vieira PJC, Teixeira PJZ, Silva FA, Riberio JP and Berton DC. Exercise capacity in adolescent and adult patients with Post-infectious bronchiolitis obliterans. Pediatr Pulmonol. 2014; 49:911-918.

2. Murtagh P, Giubergia V, Viale D, Bauer G and Pena HG. Lower respiratory infections by adenovirus in children. Clinical features and risk factors for bronchiolitis obliterans and mortality. Pediatr Pulmonol. 2009; 44:450-456.

3. Castro-Rodriguez JA, Giubergia V, Fischer GB, Castanos C, Sarria EE, Gonzalez R, Mattiello R, VegaBriceno LE and Murtagh P. Post-infectious bronchiolitis obliterans in children: the South america contribution. Acta Pediatrica. 2014; 103:913-921.

4. Mauad T, Schadewijk A, Schrumpf J, Hack CE, Fernezlian S, Garippo AL, Ejzenberg B, Hiemstra PS, Rabe KF and Dolhnikoff M. Lymphocytic inflammation in childhood bronchiolitis obliterans. Pediatr Pulmonol. 2004; 38:233-239.

5. Moonnumakal SP and Fan LL. Bronchiolitis obliterans in children. Curr Opin Pediatr. 2008; 20:272-278.

6. Colom AJ and Teper AM. Clinical prediction rule to diagnose Post-infectious bronchiolitis obliterans in children. Pediatr Pulmonol. 2009; 44:1065-1069.

7. Fischer GB, Sarria EE, Mattielo R, Mocelin HT and Castro-Rodriguez JA. Paediatr Respir Rev. 2010; 11:233-239.

8. Colom AJ and Teper AM. Post-infectious bronchiolitis obliterans. Pediatr Pulmonol. 2019; 54:212-219.

9. Jerkic SP, Brinkmann F, Calder A, Casey A, Dishp M, Griese M, Kurland G, Niemitz M, Nyilas S, Schramm D, Schubert R, Tamm M, Zielen S and Rosewich M. Post-infectious bronchiolitis obliterans in children: Diagnostic workup and therapeutic options: A workshop report. Can Respir J. 2020; 5852827.

10. Li YN, Liu L, Qiao HM, Cheng H and Cheng HJ. Post-infectious bronchiolitis obliterans in childeren: a review of 42 cases. BMC Pediatr. 2014; 14:238. 
11. Kim CK, Kim SW, Kim JS, Koh YY, Cohen AH, Deterding RR and White CW. Bronchiolitis obliterans in the 1990s in Korea and the United States. Chest; 2001:1101-1106.

12. Giubergia V, Salim M, Fraga J, Castiglioni N, Sen L, Castanos C and Mangano A. Post-infectious bronchiolitis obliterans and mannose binding lectin insufficiency in Argentinean childeren. Respirology. 2015; 20:982-986.

13. Bandeira T, Negreiro F, Ferreira R, Salgueiro M, Lobo L, Aguiar P and Trindada JC. Clinical, radiological and physiological differences between obliterative bronchiolitis and problematic severe asthma in adolescents and young adults. Pediatr Pulmonol. 2011; 46:573-580.

14. Jang YY, Park HY and Chang HL. Serum YKL-40 levels may help distinguish exacerbation of Postinfectious bronchiolitis obliterans from acute bronchiolitis in young childeren. Eur J Pediatr. 2017; 176:971978.

15. Xie BQ, Wang W, Zhang WQ, Guo XA, Yang MF, Wang L, He ZX and Tian YQ. Ventilationperfursion scintigraphy in childeren with Post-infectious bronchiolitis obliterans: A pilot study. PLoS One. 2014; 5:e98381.

16. Chang AB, Masel JP and Masters B. Post-infectious bronchiolitis obliterans: clinical, radiological and pulmonary function sequelae. Pediatr Radiol. 1998; 28:23-29.

17. Yoo Y, Yu J, Kim DK, Choi SH, Kim CK and Koh YY. Methacoline and adenosine 5' monophosphate challenges in childeren with Post-infectious bronchiolitis obliterans. Eur Respir J. 2006; 27:36-41.

18. Kim J, Kim MJ, Sol IS, Sohn MH, Yoon H, Shin HJ, Kim KW and Li MJ. Quantitative CT and pulmonary function in childeren with Post-infectious bronchiolitis obliterans. PLoS One. 2019; 4:e0214647.

19. Wang X, Liu C, Wang M, Zhang Y, Li H and Liu G. Clinical features of Post-infectious bronchiolitis obliterans in childeren undergoing long-term azithromycin treatment. Exp Ther Med. 2015; 6:2379-2383.

20. Onay ZR, Gursoy TR, Aslan AT, Eyuboglu TS, Kibar BS, Pekcan S, Hangul M, Kose M, Budakoglu II, Gokturk B. Post-infectious bronchiolitis obliterans masked by misdiognosis as asthma. Pediatr Pulmonol. 2020; 55:1007-1011.

21. Castro-rodriguez JA, Daszenies C, Garcia M, Meyer R and Gonzales R. Adenovirus pneumonia in infants and factors for developing bronchiolitis obliterans: A 5 years follow up. Pediatr Pulmonol. 2006; 41:957-953.

22. Zhang L, Irion K, Kozakewich H, Reid L, Camargo JJ, Porto NS and Silva FA. Clinical course of postinfectious bronchiolitis obliterans. Pediatr Pulmonol. 2000; 29:341:350.

23. Colom AJ, Teper AM, Vollmer WM and Diette GB. Risk factors fort he development of bronchiolitis obliterans in childeren with bronchiolitis. Thorax. 2006; 61:503-506.

24. Lino CA, Batista AKM, Soares MAD, Freitas AEH, Gomes LC, Filho JHM and Gomes VCC. Bronchiolitis obliterans: clinical and radiological profile of childeren followed-up in a reference outpatient clinic. Rev Paul Pediatr. 2013; 1:10-16.

25. Sarria EE, Mundstock E, Machado DG, Mocelin HT, Fischer GB, Furlan SP, Antonello ICF, Stein R and Mattiello R. Health-related quality of life in patients with bronchiolitis obliterans. J Pediatr. 2018; 4:374-379.

26. Kavaliunaite E and Aurora P. Diagnosing and managing bronchiolitis obliterans in childeren. Expert Rev Respir Med. 2019; 5:481-488.

27. Zhang XM, Lu AZ, Yang HW, Qian LL, Wang LB and Zhang XB. Clinical features of Post-infectious bronchiolitis obliterans in childeren undergoing long-term nebulization treatment. 2018; 14:498-503.

28. Cazzato S, Poletti V, Bernardi F, Loroni L, Bertelli L, Colonna S, Zapulla F, Timoncini G and Cicognani A. Airway inflammation and lung function decline in childhood Post-infectious bronchiolitis obliterans. Pediatr Pulmonol. 2008; 43:381-390. 
29. Tomikawa SO, Adde FV, da Silva Filho LV, Leone C and Rodrigues JC. Follow up on pediatric patients with bronchiolitis obliterans treated with corticosteroid pulse therapy. Orphanet J Rare Dis. 2014;9:128.

30. Yuksel H, Yilmaz O, Urk V, Yuksel D, Goktan C, Savas R and Sayit E. Clinical significance of lung perfusion defects in children with post-infectious bronchilitis obliterans. Tuber Toraks. 2009; 4:376-382.

31. Mattiello R, SarriaEE, Mallol J, Fischer GB, Mocelin H, Bello R, Flores JAM, Irion K and Jones Y. Pediatr Pulmonol. 2010; 45:315-319.

32. Rosewich M, Zissler UM, Kheiri T, Voss S, Eickmeier O, Schulze J, Herrmann E, Dücker RP, Schubert R and Zielen S.Airway inflammation in childeren and adolescents with bronchilitis obliterans. Cytokine 2015; $73: 156-162$.

33. Mosquera RA, Hashmi SS, Pacheo SE, Reverdin A, Chevallier J and Colasurdo. Dysanaptic growth of lung and airway in childeren with post-infectious bronchiolitis obliterans. Clin Respir J. 2014; 1:64-71.

34. Yalcin E, Doğru D, Haliloğlu M, Ozcelik U, Kiper N and Gocmen A. Post-infectious bronchiolitis obliterans in children: clinical and radiological profile and prognostic factors. Respiration;2003; 70:371-375

35. Aguerre V, Castanos C, Pena HG, Grenoville M and Murtagh P. Post-infectious bronchiolitis obliterans in childeren: Clinical and pulmonary function findings. Pediatr Pulmonol. 2010; 45:1180-1185.

\section{Hosted file}

Tables.pdf available at https://authorea.com/users/367172/articles/486680-post-infectiousbronchiolitis-obliterans-in-children-clinical-and-radiological-evaluation-and-lon-termresults

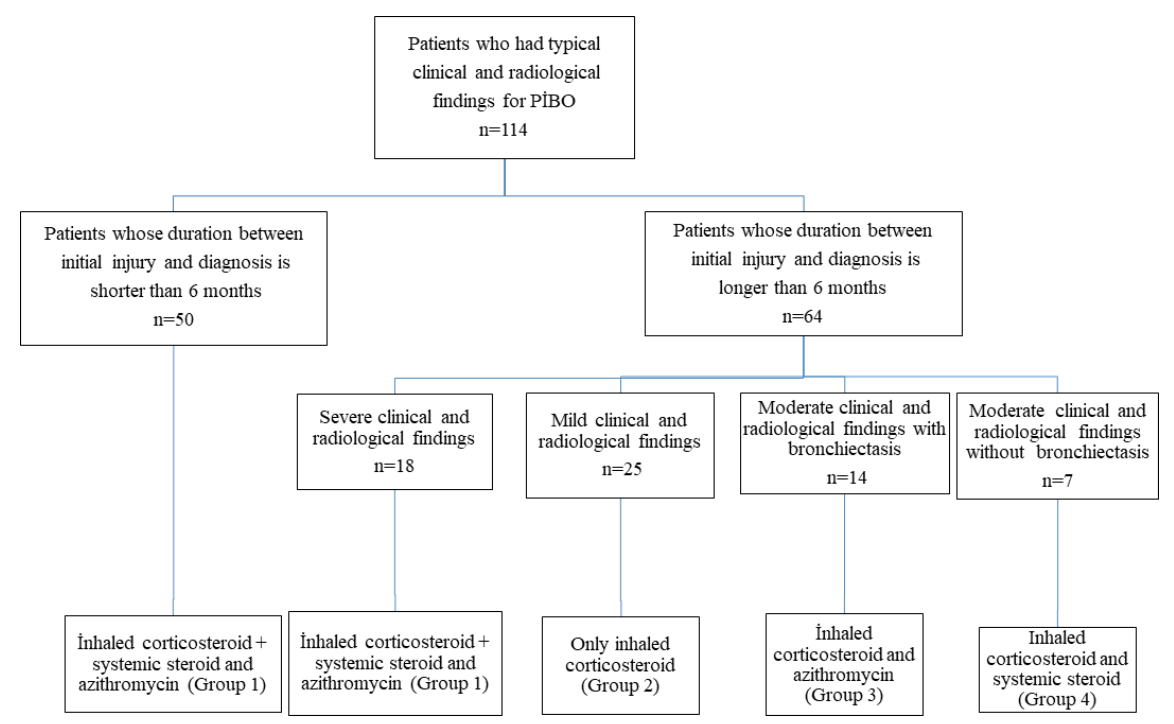

